\title{
Bohr-Sommerfeld tori and relative Poincaré series on a complex hyperbolic space
}

\author{
TATYANA FOTH
}

Let $\Gamma$ be a cocompact discrete subgroup of $S U(n, 1)$ which acts freely on $B^{n}=S U(n, 1) / U(n)$. We suggest a construction of relative Poincaré series associated to loxodromic elements of $\Gamma$. For $\Gamma \subset S U(2,1)$ we describe Bohr-Sommerfeld tori in $\Gamma \backslash B^{2}$ associated to hyperbolic elements of $\Gamma$ and compute the asymptotics of the relative Poincaré series associated to hyperbolic elements of $\Gamma$ in semi-classical limit.

\section{Introduction.}

\subsection{General definitions.}

We shall start with a brief review of the general concept of an automorphic form. Let $G$ be a connected non-compact real semi-simple Lie group with a finite center, which we also assume to be unimodular, $K$ be a maximal compact subgroup of $G, \Gamma$ be a discrete subgroup of $G$. Let $V$ be a finitedimensional vector space, $\rho: K \rightarrow G L(V)$ be an (anti)-representation of $\mathrm{K}$. A smooth $Z(\mathfrak{g})$-finite function $f: G \rightarrow V$ is called an automorphic form on $\mathbf{G}$ for $\Gamma$ if

$$
f(\gamma g k)=f(g) \rho(k)
$$

for any $\gamma \in \Gamma, g \in G, k \in K$, and there are a positive constant $C$ and a non-negative integer $m$ such that

$$
|f(g)| \leq C\|g\|^{m}
$$

for any $g \in G$, here $|$.$| is a norm in V$, and $\|g\|^{2}=\operatorname{tr}\left(g^{*} g\right)$ is taken in the adjoint representation of $G$.

The automorphy law (1) means geometrically that $f$ defines a $\Gamma$-invariant section of the vector bundle $G \times_{K} V \rightarrow G / K$ associated to the principal bundle $G \rightarrow G / K$, where $G \times{ }_{K} V=G \times V / \sim$, and the equivalence relation is given by $(g k, v) \sim(g, v \rho(k))$. 
The growth condition (2) is automatically satisfied with $m=0$ in the case when $\Gamma \backslash G$ is compact.

Recall also that a function $f: G \rightarrow V$ is said to be $\mathbf{Z}(\mathfrak{g})$-finite if it is annihilated by an ideal $I$ of $Z(\mathfrak{g})$ of a finite codimension, here $Z(\mathfrak{g})$ is the center of the universal enveloping algebra $U(\mathfrak{g})$ (over $\mathbb{C}$ ). $U(\mathfrak{g})$ can be identified with the algebra $D(G)$ of all left-invariant differential operators on $G$ (with complex coefficients): to $Y \in \mathfrak{g}$ is associated a differential operator $Y f(g)=\left.\frac{d}{d t} f\left(g e^{t Y}\right)\right|_{t=0}$, this establishes a linear map $\mathfrak{g} \rightarrow D(G)$ which extends to an isomorphism from $U(\mathfrak{g})$ onto $D(G)$. $Z(\mathfrak{g})$ can be viewed as the subalgebra of all bi-invariant differential operators, it is isomorphic to a polynomial ring in $l$ letters where $l$ is the rank of $G$. A useful example to have in mind is $G=S L(2, R)$ and $\operatorname{codim} I=1$, then we have: $l=1$, $Z(\mathfrak{g})$ is generated by the Casimir operator $\mathcal{C}$, and saying that a function $f$ is $Z(\mathfrak{g})$-finite is equivalent to stating that $f$ is an eigenfunction of $\mathcal{C}$.

A well-known construction of an automorphic form on $G$ is Poincaré series

$$
\sum_{\gamma \in \Gamma} q(\gamma g),
$$

where the function $q: G \rightarrow V$ is $Z(\mathfrak{g})$-finite and $K$-finite on the right (i.e., the set of its right translates under elements of $K$ is a finite-dimensional vector space), and $q \in L^{1}(G)$. One can also consider relative Poincaré series

$$
\sum_{\gamma \in \Gamma_{0} \backslash \Gamma} q(\gamma g),
$$

where $q: G \rightarrow V$ is $Z(\mathfrak{g})$-finite, $K$-finite on the right, $\Gamma_{0}$-invariant, and $q \in L^{1}\left(\Gamma_{0} \backslash G\right)$.

Let us explain now how to construct an automorphic form on $G / K$. An automorphy factor is a map $\mu: \Gamma \times G / K \rightarrow G L(V)$ such that $\mu\left(g_{1} g_{2}, x\right)=$ $\mu\left(g_{1}, g_{2} x\right) \mu\left(g_{2}, x\right)$. It allows to define an automorphic form on $\mathbf{G} / \mathbf{K}$ as a function $f: G / K \rightarrow V$ such that

$$
f(\gamma x) \mu(\gamma, x)=f(x)
$$

for any $\gamma \in \Gamma, x \in G / K$. Notice that then the function

$$
F(g)=f(g(0)) \mu(g, 0),
$$

where $g \in G, x=g(0) \in G / K$, satisfies (1) with $\rho(k)=\mu(k, 0)$, where 0 is the fixed point of $K$ in $G / K$. If $f$ is holomorphic then $F$ is $Z(\mathfrak{g})$-finite. 
In particular, for a smooth function $q \in L^{1}(G / K)$ the Poincaré series on $G / K$ is

$$
\sum_{\gamma \in \Gamma} q(\gamma x) \mu(\gamma, x)
$$

\subsection{Automorphic forms on bounded symmetric domains and quantization.}

Consider a classical system $(M, \omega)$, where $M$ is a manifold, and $\omega$ is a symplectic form on $M$. The main problem of quantization is to associate a quantum system $(\mathcal{H}, \mathcal{O})$ to $(M, \omega)$, where $\mathcal{H}$ is a Hilbert space and $\mathcal{O}$ consists of symmetric operators with domain in $\mathcal{H}$. Elements of $\mathcal{H}$ are wave functions (quantum-mechanical states), and elements of $\mathcal{O}$ are quantum observables.

The map $f \mapsto \hat{f}$, where $f \in C^{\infty}(M)$ and $\hat{f} \in \mathcal{O}$, should satisfy the following requirements:

1) it is $\mathbb{R}$-linear,

2) if $f=$ const then $\hat{f}$ is the corresponding multiplication operator,

3) if $\left\{f_{1}, f_{2}\right\}=f_{3}$ then $\hat{f}_{1} \hat{f}_{2}-\hat{f}_{2} \hat{f}_{1}=i \hbar \hat{f}_{3}$.

These are Dirac quantization conditions and they are famously impossible to satisfy in most cases, so one should consider a certain modification of them.

How do automorphic forms appear in the context of quantization?

Suppose that $M$ is a compact Kähler manifold of complex dimension $n$ which is a quotient of a bounded symmetric domain $D=G / K$ by the action of a discrete subgroup $\Gamma$, i.e. $M=\Gamma \backslash D$. Then $\mathcal{H}$ consists of holomorphic automorphic forms on $D$ for $\Gamma$. More precisely, let us consider the well-known quantization scheme for compact Kähler manifolds via Toeplitz operators (it is related to the standard scheme of geometric quantization with Kähler polarization). Automorphic forms are holomorphic sections of $L^{\otimes k}$, where the canonical line bundle $L=\Lambda^{n} T_{\text {hol }}^{*} M$ is the quantizing line bundle on $M$, here $k$ is a positive integer which determines the weight of an automorphic form, and $\hbar=\frac{1}{k}$.

We also notice that the automorphic form (3) is a sum of coherent states associated to a holomorphic discrete series representation of $G$.

Let us describe all this in a bit more details. Let $D=G / K$ be a bounded symmetric domain, it is a Hermitian symmetric space of noncompact type. The irreducible Hermitian spaces of non-compact type are

I) $S U(p, q) / S(U(p) \times U(q))$,

II) $S p(p, \mathbb{R}) / U(p)$, 
III) $S O^{*}(2 p) / U(p)$,

IV) $S O_{o}(p, 2) / S O(p) \times S O(2)$

(and also there is the case of an exceptional Lie group). We have a metric

$$
d s^{2}=g_{i j} d z^{i} d \bar{z}^{j},
$$

the corresponding Kähler form is $\omega=i g_{i j} d z^{i} \wedge d \bar{z}^{j}=i \partial \bar{\partial} \ln K(z, z)$, where $K(z, w)$ is the Bergman kernel of the domain $D$. Recall that $K(z, w)=$ $\overline{K(w, z)}$ and

$$
K(\gamma z, \gamma w)=[\operatorname{det} J(\gamma, z)]^{-1}[\operatorname{det} \bar{J}(\gamma, w)]^{-1} K(z, w) .
$$

A quantizing line bundle $L \rightarrow M=\Gamma \backslash D$ is defined as a line bundle such that the curvature of its natural connection is the Kähler form $\omega$ on $M$. Denoting the canonical line bundle by $L$ we see that the potential 1 -form corresponding to the natural connection on $L$ is $\theta=i \partial \ln (s, s)=$ $-i \partial \ln K(z, z)$, hence the curvature $d \theta=-i \bar{\partial} \partial \ln K(z, z)=\omega$ and this is indeed a quantizing line bundle for $M$.

A holomorphic function $f: D \rightarrow \mathbb{C}$ is called an automorphic form of weight $\mathbf{k}$ if

$$
f(\gamma z)[\operatorname{det} J(\gamma, z)]^{k}=f(z)
$$

for any $z \in D, \gamma \in \Gamma$; here $J(\gamma, z)$ is the Jacobi matrix of transformation $\gamma$ at point $z$. In the context of 1.1 the automorphy factor $\mu(\gamma, z)=[\operatorname{det} J(\gamma, z)]^{k}$. The space of automorphic forms of weight $k$ can be identified with the complex inner product space $H^{0}\left(M, L^{\otimes k}\right)$ of holomorphic sections of $L^{\otimes k}$.

Now we consider a family of maps $p_{k}$, here $k$ is a positive integer, such that $p_{k}(f)=T_{f}^{(k)}$, where $f$ belongs to the Poisson algebra of smooth realvalued functions on $M$ and $T_{f}^{(k)}$ is the Toeplitz operator on $H^{0}\left(M, L^{\otimes k}\right)$ obtained from multiplication operator $M_{f}^{(k)}(g)=f g$ on $L^{2}\left(M, L^{\otimes k}\right)$ by the orthogonal compression to the closed subspace $H^{0}\left(M, L^{\otimes k}\right)$, i.e. $T_{f}^{(k)}=$ $\Pi^{(k)} \circ M_{f}^{(k)} \circ \Pi^{(k)}$, where $\Pi^{(k)}$ is the orthogonal projection from $L^{2}\left(M, L^{\otimes k}\right)$ to $H^{0}\left(M, L^{\otimes k}\right)$.

In the Berezin scheme of quantization [1], [18] for each $\hbar=\frac{1}{k}$ we consider the space $\mathcal{F}_{\hbar}$ of functions holomorphic in $D$ and satisfying (5) with the scalar product defined by

$$
(f, g)=\operatorname{const}(\hbar) \int_{M} f(z) \bar{g}(z)[K(z, z)]^{-\frac{1}{\hbar}} d \mu(z),
$$


where $d \mu(z)=\omega^{n}$ is the $G$-invariant volume form on $D$ corresponding to the metric (4). It is clear that $\mathcal{F}_{\hbar}$ is naturally identified with $H^{0}\left(M, L^{\otimes k}\right)$. For the sake of completeness let us also explain briefly how the operator $\hat{A}$ corresponding a classical observable $A=A(z)$, is defined. First, we consider an analytic continuation $A(z, w)$ of the function $A(z)$ to $D \times D$. The covariant symbol $A(z, z)$ of $\hat{A}$ is defined as the diagonal value of the function

$$
A(z, w)=\frac{\int_{M} \hat{A}\left[(K(u, w))^{\frac{1}{\hbar}}\right](K(z, u))^{\frac{1}{\hbar}} d \mu(u)}{\int_{M}(K(u, w))^{\frac{1}{\hbar}}(K(z, u))^{\frac{1}{\hbar}} d \mu(u)}
$$

and

$$
(\hat{A} f)(z)=\operatorname{const}(\hbar) \int_{M} A(z, w) f(w)[K(z, w)]^{\frac{1}{\hbar}}[K(w, w)]^{-\frac{1}{\hbar}} d \mu(w) .
$$

So we end up with the algebra $A_{\hbar}$ of covariant symbols of bounded operators acting in $\mathcal{F}_{\hbar}$. The $*$-product in $A_{\hbar}$ is given by

$$
A_{1} * A_{2}(z, z)=\operatorname{const}(\hbar) \int_{M} A_{1}(z, w) A_{2}(w, z)\left(\frac{K(z, w) K(w, z)}{K(z, z) K(w, w)}\right)^{\frac{1}{\hbar}} d \mu(w) .
$$

In conclusion let us discuss the Poincaré series (3). Consider a unitary representation of $G$ in $L^{2}(G / K)$ given by the operators

$$
\left[\pi^{k}(g)(q)\right](z)=[\operatorname{det} J(g, z)]^{k} q(g z) .
$$

It can be regarded as a subrepresentation of the right regular representation of $G$ in $L^{2}(\Gamma \backslash G)$. Fix $q \in \mathcal{F}_{\hbar}$, then the set $\left\{\pi^{k}(g)(q) \mid g \in G\right\}$ is a system of (generalized) coherent states. Strictly speaking, we should regard two coherent states $\pi^{k}\left(g_{1}\right)(q)$ and $\pi^{k}\left(g_{2}\right)(q)$ as equivalent if $\pi^{k}\left(g_{1}\right)(q)=e^{i \alpha} \pi^{k}\left(g_{2}\right)(q)$. Now it is clear that (3) is a sum of coherent states which belong to the subsystem associated to $\Gamma$.

\subsection{Comments on the subject of the present paper.}

In [9] and in the present paper we consider holomorphic automorphic forms on $D=\mathbb{H}_{\mathbb{C}}^{n}=S U(n, 1) / U(n)$. In [9] we construct sets of relative Poincaré series which span the spaces of $\mathbb{C}$-valued holomorphic cusp forms on a finite volume quotient of $D$. In the present paper we regard holomorphic $\mathbb{C}$ valued automorphic forms on $\mathbb{H}_{\mathbb{C}}^{n}$ as holomorphic sections of the line bundle $L^{\otimes k} \rightarrow \Gamma \backslash \mathbb{H}_{\mathbb{C}}^{n}$, where $L$ is a quantizing line bundle on $\Gamma \backslash \mathbb{H}_{\mathbb{C}}^{n}, k$ is an integer, and $\Gamma$ is a discrete cocompact subgroup of $S U(n, 1)$. We construct relative 
Poincaré series associated to loxodromic elements of $\Gamma$ and we address an interesting problem which is not resolved for Poincaré series in general: is it true that these series are not identically zero ? We restrict ourselves to the case of complex dimension 2 and answer "yes" to this question going through the following steps: 1 ) to each hyperbolic element of $\Gamma$ we associate a sequence $\Lambda(l), l \geq 1$, of Legendrian submanifolds of the unit circle bundle in $L^{*}$ such that the corresponding Lagrangian tori in $\Gamma \backslash S U(2,1) / U(2)$ satisfy a Bohr-Sommerfeld condition, 2) following the method of [6] we compute the $k$-th component $u_{k}$ of the delta-function associated to $\Lambda(l)$ and the leading order asymptotics of $\left\|u_{k}\right\|$, which allows us to conclude that the relative Poincaré series associated to hyperbolic elements are not zero for large values of $k$ (i.e. in semi-classical limit $\hbar=\frac{1}{k} \rightarrow 0$ ).

\section{Preliminaries.}

\subsection{Complex hyperbolic space.}

Consider the complex hyperbolic space

$$
\mathbb{H}_{\mathbb{C}}^{n}=S U(n, 1) / S(U(n) \times U(1))=\mathbb{P}\left(\left\{z \in \mathbb{C}^{n+1} \mid\langle z, z\rangle<0\right\}\right) \simeq B^{n},
$$

here $B^{n}$ is the open unit ball in $\mathbb{C}^{n},\langle.,$.$\rangle is the Hermitian product on$ $\mathbb{C}^{n+1}$ given by $\langle z, w\rangle=z_{1} \bar{w}_{1}+\cdots+z_{n} \bar{w}_{n}-z_{n+1} \bar{w}_{n+1}$.

A vector $z \in \mathbb{C}^{n+1}-\{0\}$ is called negative (resp. null, positive) if the value of $\langle z, z\rangle$ is negative (resp. null, positive).

For $z, w \in B^{n}$ the corresponding vectors in $\mathbb{C}^{n+1}$ are $\left(\begin{array}{c}z \\ 1\end{array}\right)=\left(\begin{array}{c}z_{1} \\ \cdots \\ z_{n} \\ 1\end{array}\right)$ and $\left(\begin{array}{c}w \\ 1\end{array}\right)=\left(\begin{array}{c}w_{1} \\ \cdots \\ w_{n} \\ 1\end{array}\right)$, and we denote $\langle z, w\rangle=z_{1} \bar{w}_{1}+\cdots+z_{n} \bar{w}_{n}-1$.

The group of isometries of $\mathbb{H}_{\mathbb{C}}^{n}$ is $P U(n, 1)=S U(n, 1) /$ center. The group $S U(n, 1)$ acts on $B^{n}$ and on the boundary sphere $\partial B^{n}=\mathbb{P}(\{z \in$ $\left.\left.\mathbb{C}^{n+1}-\{0\} \mid\langle z, z\rangle=0\right\}\right)$ by fractional-linear transformations: for

$$
\gamma=\left(\begin{array}{cccc}
a_{11} & \ldots & a_{1 n} & b_{1} \\
\ldots & \ldots & \ldots & \ldots \\
a_{n 1} & \ldots & a_{n n} & b_{n} \\
c_{1} & \ldots & c_{n} & d
\end{array}\right)
$$


and $z \in B^{n}$ (or $z \in \partial B^{n}$ ) we have:

$$
\gamma z=\left(\frac{a_{11} z_{1}+\cdots+a_{1 n} z_{n}+b_{1}}{c_{1} z_{1}+\cdots+c_{n} z_{n}+d}, \ldots, \frac{a_{n 1} z_{1}+\cdots+a_{n n} z_{n}+b_{n}}{c_{1} z_{1}+\cdots+c_{n} z_{n}+d}\right)^{T},
$$

and

$$
\operatorname{det} J(\gamma, z)=\left(c_{1} z_{1}+\cdots+c_{n} z_{n}+d\right)^{-(n+1)},
$$

where $J(\gamma, z)$ denotes the Jacobi matrix of transformation $\gamma$ at point $z$.

An automorphism is called loxodromic if it has no fixed points in $B^{n}$ and fixes two points in $\partial B^{n}$. Notice that the fixed points of the automorphisms correspond to the eigenvectors of the corresponding matrices in $U(n, 1)$. A loxodromic automorphism is called hyperbolic if it has a lift to $U(n, 1)$ all of whose eigenvalues are real.

A loxodromic element $\gamma_{0} \in S U(n, 1)$ has $n-1$ positive eigenvectors and two null eigenvectors.

Let $v_{1}, \ldots, v_{n-1}$ be the positive eigenvectors of $\gamma_{0}$ and $\tau_{1}, \ldots, \tau_{n-1}$ be the corresponding eigenvalues. Then $\left|\tau_{j}\right|=1,1 \leq j \leq n-1$.

Let $X, Y$ be the null eigenvectors of $\gamma_{0}$. Then the corresponding eigenvalues are $\lambda$ and $\bar{\lambda}^{-1}$ for some $\lambda \in \mathbb{C},|\lambda|>1$.

A loxodromic transformation can always be represented by a matrix in $U(n, 1)$ with eigenvalues $\tau_{1}, \ldots, \tau_{n-1}, \lambda, \lambda^{-1}$ where $\lambda \in \mathbb{R},|\lambda|>1$.

The geodesic connecting $X$ and $Y$ is the geodesic in the Poincaré metric on the complex line containing $X$ and $Y$ (so it is an arc of a circle orthogonal to $\partial B^{n}$ or a diameter), it is $\gamma_{0}$-invariant and is called the axis of $\gamma_{0}$.

\subsection{Automorphic forms and geometry of the quotient.}

Consider a compact manifold $X:=\Gamma \backslash B^{n}$, where $\Gamma$ is a discrete cocompact subgroup of $S U(n, 1)$ which acts freely on $B^{n}$.

The Bergman kernel for the domain $B^{n}$ is $K(z, w)=\frac{1}{(-\langle z, w\rangle)^{n+1}}$ (up to a multiplicative constant) and an $S U(n, 1)$-invariant Kähler form on $B^{n}$ is

$$
\Omega=i \partial \bar{\partial} \ln K(z, z)=-\frac{(n+1) i}{\langle z, z\rangle^{2}}\left(\langle z, z\rangle \sum_{j=1}^{n} d z_{j} \wedge d \bar{z}_{j}-\langle d z, z\rangle \wedge\langle z, d z\rangle\right)
$$

Remark 2.1. With this normalization the holomorphic sectional curvature is equal to $-\frac{4}{n+1}$ and the sectional curvature is pinched between $-\frac{4}{n+1}$ and $-\frac{1}{n+1}$. 
A holomorphic function $f: B^{n} \rightarrow \mathbb{C}$ satisfying the automorphy law

$$
f(\gamma z)(\operatorname{det} J(\gamma, z))^{k}=f(z)
$$

for any $\gamma \in \Gamma$ is called an automorphic form of weight $(n+1) k$ for $\Gamma$. The corresponding automorphic form on $S U(n, 1)$ is given by $F(g)=f(g(0)) \zeta^{k}$, where $\zeta=\zeta(g)=\operatorname{det} J(g, 0)$ and the origin 0 of $B^{n}$ is the fixed point of $K=S(U(n) \times U(1)) \simeq U(n)$. The automorphy law on the group is

$$
F(\gamma g \kappa)=F(g) \rho(\kappa),
$$

where $\rho(\kappa)=(\operatorname{det} J(\kappa, 0))^{k}$, for any $g \in G, \gamma \in \Gamma, \kappa \in K$. Notice that $\gamma: \zeta \rightarrow \zeta \operatorname{det} J(\gamma, z)$ for any $\gamma \in S U(n, 1)$. The automorphic form $F(g)$ can be regarded as a section of $L^{k}$ where $L=\Lambda^{n} T_{\text {hol }}^{*} X$ is the canonical line bundle, and $T_{\text {hol }}^{*} X$ denotes the holomorphic cotangent bundle on $X$.

We shall denote the space of automorphic forms of weight $(n+1) k$ for $\Gamma$ on $B^{n}$ by $S_{(n+1) k}(\Gamma)$ and the corresponding space of automorphic forms on $S U(n, 1)$ by $\tilde{S}_{(n+1) k}(\Gamma)$. The inner product in each of these spaces is given by

$$
(f, g)=\left(f(z) \zeta^{k}, g(z) \zeta^{k}\right)=\int_{\Gamma \backslash B^{n}} f \bar{g}(-\langle z, z\rangle)^{(n+1) k} d V,
$$

where

$$
d V=i^{n} \frac{d z_{1} \wedge d \bar{z}_{1} \wedge \cdots \wedge d z_{n} \wedge d \bar{z}_{n}}{(-\langle z, z\rangle)^{n+1}}
$$

is a constant multiple of the $S U(n, 1)$-invariant volume form for the metric corresponding to $\Omega$.

Given a subgroup $\Gamma_{0}$ of $\Gamma$ and a holomorphic function $q(z)$ satisfying (6) for all $\gamma \in \Gamma_{0}$ and such that $\int_{\Gamma_{0} \backslash B^{n}}|q(z)|(-\langle z, z\rangle)^{\frac{(n+1) k}{2}} d V<\infty$, the relative Poincaré series associated to $\Gamma_{0}$ is defined as

$$
\Theta(z)=\sum_{\gamma \in \Gamma_{0} \backslash \Gamma} q(\gamma z)(\operatorname{det} J(\gamma, z))^{k} .
$$

By Theorem A.1 (Appendix) this series converges absolutely and uniformly on compact sets and belongs to the space $S_{(n+1) k}(\Gamma)$.

The potential 1-form $\theta$ on $L^{*}$ is characterized by

$$
\nabla s=-i \theta s,
$$

where $\nabla$ is a connection on $L^{*}$ and $s$ is the unit section. In local coordinates the potential 1-form corresponding to the natural connection on $L^{*}$ is

$$
\theta=i \partial \ln (s, s)=-i \partial \ln (-\langle z, z\rangle)^{n+1} .
$$


The curvature on $L^{*}$ is $d \theta=-\Omega$, hence $L$ is the natural quantizing line bundle for $X$.

Let

$$
E_{k}=H^{0}\left(X, L^{\otimes k}\right)
$$

be the complex inner product space of holomorphic sections of the $k$-th tensor power of $L$. Consider the unit circle bundle $P \subset L^{*}$. Denote also $\tilde{L}=\bigwedge^{n} T_{\text {hol }}^{*} B^{n}, \tilde{P}$ - the unit circle bundle in $\tilde{L}^{*}$.

The connection form $\alpha: T P \rightarrow \mathbb{R}$ on $P$ is

$$
\alpha=\theta+i \frac{d \zeta}{\zeta}=-(n+1) i \frac{\langle d z, z\rangle}{\langle z, z\rangle}+i \frac{d \zeta}{\zeta}=-d \psi+\frac{n+1}{2} i \frac{\langle z, d z\rangle-\langle d z, z\rangle}{\langle z, z\rangle}
$$

in local coordinates $z \in B^{n}, \zeta=(-\langle z, z\rangle)^{\frac{n+1}{2}} e^{i \psi}$. It serves as a contact form on $\tilde{P}$ and $P$.

A Lagrangian submanifold $\Lambda_{0} \subset X$ satisfies a Bohr-Sommerfeld condition if

$$
\frac{k}{2 \pi} \int_{C} \theta \in \mathbb{Z}
$$

for any closed curve $C \subset \Lambda_{0}$. The constant $\frac{1}{k}$ plays role of the Planck constant.

The unit disk bundle in $L^{*}$ is a compact, strictly pseudoconvex domain with smooth boundary $P$. Let us consider the Hardy space of $P: E \subset L^{2}(P)$ and the Szëgo projector

$$
\Pi: L^{2}(P) \rightarrow E
$$

given by the orthogonal projection of $L^{2}(P)$ onto $E$. We identify:

$$
E=\oplus_{k=0}^{\infty} E_{k} .
$$

We also denote

$$
\begin{aligned}
& \tilde{E}_{k}=\left\{f(z) \zeta^{k} \mid(z, \zeta) \in \tilde{P}, f \text { is holomorphic on } B^{n},\right. \\
& \left.\qquad \int_{B^{n}}|f(z)|^{2}(-\langle z, z\rangle)^{(n+1) k} d V<\infty\right\} .
\end{aligned}
$$

We shall denote the corresponding orthogonal projection by

$$
\tilde{\Pi}: L^{2}(\tilde{P}) \rightarrow \oplus_{k=0}^{\infty} \tilde{E}_{k} .
$$

Both projectors can be extended to a class of distributions including the delta function. 


\section{Construction of relative Poincaré series associated to certain loxodromic elements of $\Gamma$.}

Consider a loxodromic automorphism of $B^{n}$, represent it by a matrix $\gamma_{0} \in U(n, 1)$ with eigenvalues $\tau_{1}, \ldots, \tau_{n-1}, \lambda, \lambda^{-1},\left|\tau_{j}\right|=1, j=1, \ldots, n-1$, $\lambda \in \mathbb{R},|\lambda|>1$, denote the corresponding eigenvectors by $v_{1}, \ldots, v_{n-1}, X, Y$ $\left(v_{1}, \ldots, v_{n-1}\right.$ are positive, $X, Y$ are null). Notice that if each $\tau_{j}$ is a root of 1 then some power of $\gamma_{0}$ is a hyperbolic element.

Now consider an arbitrary loxodromic element $\gamma_{0} \in \Gamma \subset S U(n, 1)$ represented as described above and assume that $\gamma_{0}$ satisfies the following condition.

Assumption 3.1. Assume that 1 is among the eigenvalues of $\gamma_{0}$.

Remark 3.2. If $g \in U(n, 1)$ is hyperbolic then $g^{2}$ is a hyperbolic element of $S U(n, 1)$ which satisfies Assumption 3.1 and has the same eigenvectors as $g$.

Generalizing the construction suggested in [9], for any collection, w.l.o.g. $v_{1}, \ldots, v_{m}, m \leq n-1$, of positive eigenvectors corresponding to eigenvalue 1 we construct a relative Poincaré series

$$
\Theta_{\gamma_{0}, l, k}=\sum_{\gamma \in \Gamma_{0} \backslash \Gamma} q_{l}(\gamma z)(\operatorname{det} J(\gamma, z))^{2 k} \in S_{2(n+1) k}(\Gamma),
$$

where $\Gamma_{0}=\left\langle\gamma_{0}\right\rangle$,

$$
q_{l}(z)=\frac{\left\langle z, v_{1}\right\rangle^{l_{1}} \cdots\left\langle z, v_{m}\right\rangle^{l_{m}}}{(\langle z, X\rangle\langle z, Y\rangle)^{(n+1) k+\frac{l_{1}+\cdots+l_{m}}{2}}}
$$

$l_{1}, \ldots, l_{m}$ are positive integers such that $l_{1}+\cdots+l_{m}$ is even, $l=\left(l_{1}, \ldots, l_{m}\right)$. The series converges absolutely and uniformly on the compact sets of $B^{n}$ by the Theorem A.1 (Appendix) for $k \geq 1$.

If $n=2$ then the loxodromic elements of $\Gamma$ satisfying Assumption 3.1 are exactly the hyperbolic elements of $\Gamma$. The relative Poincaré series associated to a hyperbolic element $\gamma_{0} \in \Gamma$ is

$$
\Theta_{\gamma_{0}, l, k}=\sum_{\gamma \in \Gamma_{0} \backslash \Gamma} q_{l}(\gamma z)(\operatorname{det} J(\gamma, z))^{2 k} \in S_{6 k}(\Gamma),
$$

where $\Gamma_{0}=\left\langle\gamma_{0}\right\rangle$,

$$
q_{l}(z)=\frac{\langle z, v\rangle^{2 l}}{(\langle z, X\rangle\langle z, Y\rangle)^{3 k+l}}
$$

and $l$ is a non-negative integer. 
Remark 3.3. Let $\gamma_{1}$ and $\gamma_{2}$ be hyperbolic elements of $\Gamma$. If $\gamma_{1}=\gamma_{2}^{N}$ for a positive integer $N$, then $\Theta_{\gamma_{1}, l, k}=N \Theta_{\gamma_{2}, l, k}$.

Remark 3.4. In further exposition we assume that $l>0$. The relative Poincaré series with $l=0$ studied in [9] are associated to closed geodesics (not to Lagrangian tori).

\section{Bohr-Sommerfeld tori.}

Consider a hyperbolic element $\gamma_{0} \in \Gamma \subset S U(2,1)$, denote its null eigenvectors by $X=\left(\begin{array}{c}X_{1} \\ X_{2} \\ 1\end{array}\right), Y=\left(\begin{array}{c}Y_{1} \\ Y_{2} \\ 1\end{array}\right)$, denote its positive eigenvector by $v$, then the corresponding eigenvalues are $\lambda, \lambda^{-1}, 1$, for $\lambda \in \mathbb{R},|\lambda|>1$. We have:

$$
\langle v, X\rangle=\langle v, Y\rangle=\langle X, X\rangle=\langle Y, Y\rangle=0 .
$$

We normalize $v$ so that $\langle v, v\rangle=1$, then the matrix

$$
A:=\left[\begin{array}{lll}
v & \frac{X}{\langle X, Y\rangle}+\frac{Y}{2} & \frac{X}{\langle X, Y\rangle}-\frac{Y}{2}
\end{array}\right]
$$

belongs to $S U(2,1)$.

The transformation $A^{-1}=s\left(\begin{array}{c}\bar{v}^{T} \\ \frac{\bar{X}^{T}}{\langle Y, X\rangle}+\frac{\bar{Y}^{T}}{2} \\ \frac{\bar{X}^{T}}{\langle Y, X\rangle}-\frac{\bar{Y}^{T}}{2}\end{array}\right) s$, where $s=\left(\begin{array}{ccc}1 & 0 & 0 \\ 0 & 1 & 0 \\ 0 & 0 & -1\end{array}\right)$, maps the complex line containing $X$ and $Y$ to the complex line $\left\{z_{1}=0\right\}$ and maps the geodesic connecting $X$ and $Y$ to the geodesic $\tilde{C}$ connecting $(0,-1)$ and $(0,1)$. More precisely

$$
A^{-1} \cdot X=\left(\begin{array}{c}
0 \\
\frac{\langle X, Y\rangle}{2} \\
\frac{\langle X, Y\rangle}{2}
\end{array}\right), A^{-1} \cdot Y=\left(\begin{array}{c}
0 \\
1 \\
-1
\end{array}\right)
$$

where $\cdot$ stands for the standard linear action of $G L(3, \mathbb{C})$ on $\mathbb{C}^{3}$, so

$$
A^{-1} X=\left(\begin{array}{l}
0 \\
1 \\
1
\end{array}\right), A^{-1} Y=\left(\begin{array}{c}
0 \\
-1 \\
1
\end{array}\right)
$$

also $A^{-1} \cdot v=\left(\begin{array}{l}1 \\ 0 \\ 0\end{array}\right)$ 
The following loxodromic element of $S U(2,1)$ preserves $\tilde{C}$ and the complex line $\left\{z_{1}=0\right\}$ :

$$
\gamma:=A^{-1} \gamma_{0} A=\left(\begin{array}{ccc}
1 & 0 & 0 \\
0 & a & b \\
0 & b & a
\end{array}\right)
$$

where

$$
a=\frac{\lambda^{2}+1}{2 \lambda}, b=\frac{\lambda^{2}-1}{2 \lambda} .
$$

Denote

$$
w=\left(w_{1}, w_{2}\right)^{T}=A^{-1} z, w_{1}=A^{-1} z_{1}, w_{2}=A^{-1} z_{2},
$$

and apply the change of variables

$$
\begin{gathered}
w_{2}=\frac{r e^{i \phi}-i}{r e^{i \phi}+i}, w_{1}=\sqrt{1-w_{2} \bar{w}_{2}} R e^{i \Theta}, \\
0<\phi<\pi, 0<r<+\infty, 0<R<1,0 \leq \Theta<2 \pi .
\end{gathered}
$$

Proposition 4.1. Any 2-cylinder $C_{\phi, R}=\{\phi=$ const, $R=$ const $\}$ is $\gamma$ invariant.

Remark 4.2. The coordinates $(r, \phi)$ are the polar coordinates on the upperhalf plane, $(R, \Theta)$ are the polar coordinates in the unit disc, and the coordinates $(r, \Theta)$ on the cylinder $C_{\phi, R} \simeq \mathbb{R} \times S^{1}$ are the axial and the angular coordinates respectively.

Proof. Under the action of $\gamma$

$$
w_{2} \rightarrow \frac{a w_{2}+b}{b w_{2}+a}=\frac{a \frac{r e^{i \phi}-i}{r e^{i \phi}+i}+b}{b \frac{r e^{i \phi}-i}{r e^{i \phi}+i}+a}=\frac{r(a+b) e^{i \phi}-i(a-b)}{r(a+b) e^{i \phi}+i(a-b)}=\frac{r \frac{a+b}{a-b} e^{i \phi}-i}{r \frac{a+b}{a-b} e^{i \phi}+i},
$$

so

$$
r \rightarrow r \frac{a+b}{a-b}, \quad \phi \rightarrow \phi
$$

also

$$
\frac{\left|w_{1}\right|}{\sqrt{1-w_{2} \bar{w}_{2}}} \rightarrow \frac{\left|\frac{w_{1}}{b w_{2}+a}\right|}{\sqrt{1-\left|\frac{a w_{2}+b}{b w_{2}+a}\right|^{2}}}=\frac{\left|w_{1}\right|}{\sqrt{1-w_{2} \bar{w}_{2}}}
$$

so $R \rightarrow R$. 
For a positive integer $l$ consider the following submanifold of $\tilde{P}$ :

$$
\tilde{T}(l):=\left\{\left(w,(-\langle w, w\rangle)^{\frac{3}{2}} e^{i \psi}\right) \mid w \in\left\{\phi=\frac{\pi}{2}, R=\sqrt{\frac{l}{3 k+l}}\right\}, \psi=-\frac{l}{k} \Theta\right\} .
$$

The natural projection of $\tilde{T}(l)$ to $B^{2}$ is the cylinder $C_{\frac{\pi}{2}, \sqrt{\frac{l}{3 k+l}}}$ whose axis of symmetry is the geodesic $\tilde{C}$. Denote $T(l)=\langle\gamma\rangle \backslash \tilde{T}(l), \tilde{\Lambda}(l)=A \tilde{T}(l)$ and $\Gamma_{0}=\left\langle\gamma_{0}\right\rangle$.

Proposition 4.3. $\Lambda(l):=A T(l)=\Gamma_{0} \backslash \tilde{\Lambda}(l)$ is a compact Legendrian submanifold of $P$.

Proof. Both $T(l)$ and $\Lambda(l)$ are compact submanifolds of $P$ of real dimension 2.

Let us prove that $\Lambda(l)$ is Legendrian. The restriction of $\alpha$ onto $\tilde{T}(l)$ is

$$
\begin{aligned}
- & 3 i \frac{\langle d w, w\rangle}{\langle w, w\rangle}+i \frac{d \zeta}{\zeta} \\
= & -\frac{3}{2} i \frac{\bar{w}_{1} d w_{1}+\bar{w}_{2} d w_{2}}{w_{1} \bar{w}_{1}+w_{2} \bar{w}_{2}-1}+\frac{3}{2} i \frac{w_{1} d \bar{w}_{1}+w_{2} d \bar{w}_{2}}{w_{1} \bar{w}_{1}+w_{2} \bar{w}_{2}-1}-d \psi \\
= & -\frac{3}{2} i \frac{\left(1-w_{2} \bar{w}_{2}\right) R^{2} i d \Theta+\sqrt{1-w_{2} \bar{w}_{2} R^{2} d \sqrt{1-w_{2} \bar{w}_{2}}+\bar{w}_{2} d w_{2}}}{\left(1-w_{2} \bar{w}_{2}\right) R^{2}+w_{2} \bar{w}_{2}-1} \\
& +\frac{3}{2} i \frac{-\left(1-w_{2} \bar{w}_{2}\right) R^{2} i d \Theta+\sqrt{1-w_{2} \bar{w}_{2}} R^{2} d \sqrt{1-w_{2} \bar{w}_{2}}+w_{2} d \bar{w}_{2}}{\left(1-w_{2} \bar{w}_{2}\right) R^{2}+w_{2} \bar{w}_{2}-1}-d \psi \\
= & -\frac{3}{2} i \frac{\left(1-w_{2}^{2}\right) R^{2} i d \Theta+\sqrt{1-w_{2}^{2}} R^{2} d \sqrt{1-w_{2}^{2}}+w_{2} d w_{2}}{\left(1-w_{2}^{2}\right)\left(R^{2}-1\right)} \\
& +\frac{3}{2} i \frac{-\left(1-w_{2}^{2}\right) R^{2} i d \Theta+\sqrt{1-w_{2}^{2}} R^{2} d \sqrt{1-w_{2}^{2}}+w_{2} d w_{2}}{\left(1-w_{2}^{2}\right)\left(R^{2}-1\right)}-d \psi \\
= & -3 i \frac{\left(1-w_{2}^{2}\right) R^{2} i d \Theta}{\left(1-w_{2}^{2}\right)\left(R^{2}-1\right)}-d \psi=3 \frac{R^{2} d \Theta}{R^{2}-1}-d \psi \\
= & 3 \frac{\frac{l}{3 k+l} d \Theta}{\frac{l}{3 k+l}-1}+\frac{l}{k} d \Theta=0 .
\end{aligned}
$$

The form $\alpha$ is $S U(2,1)$-invariant, indeed, under the action of $M \in S U(2,1)$,

$$
(z, \zeta) \mapsto(M z, \zeta \operatorname{det} J(M, z))=\left(M z, \zeta c^{3}\right),
$$


where $c=c(z)=\left(m_{31} z_{1}+m_{32} z_{2}+m_{33}\right)^{-1}$. We have:

$$
\begin{aligned}
\alpha & =i \frac{d \zeta}{\zeta}-3 i \partial \ln (-\langle z, z\rangle) \rightarrow i \frac{d\left(c^{3} \zeta\right)}{c^{3} \zeta}-3 i \partial \ln (-\langle M z, M z\rangle) \\
& =i \frac{c^{3} d \zeta+3 c^{2} \zeta d c}{c^{3} \zeta}-3 i \partial \ln (-\langle z, z\rangle c \bar{c}) \\
& =i \frac{d \zeta}{\zeta}+3 i \frac{d c}{c}-3 i \partial \ln (-\langle z, z\rangle)-3 i \partial \ln (c \bar{c}) \\
& =i \frac{d \zeta}{\zeta}+3 i \frac{\partial c}{c}-3 i \partial \ln (-\langle z, z\rangle)-3 i \frac{\partial c}{c} \\
& =i \frac{d \zeta}{\zeta}-3 i \partial \ln (-\langle z, z\rangle) .
\end{aligned}
$$

The natural projection $\Lambda_{0}(l)$ of $\Lambda(l)$ onto $X$ is a compact Lagrangian submanifold of $X$.

Proposition 4.4. $\Lambda_{0}(l)$ satisfies a Bohr-Sommerfeld condition.

Proof. Let $\tilde{T}_{0}(l)$ be the natural projection of $\tilde{T}(l)$ onto $B^{2}$, and let $T_{0}(l)$ be the natural projection of $T(l)$ onto $X, A T_{0}(l)=\Lambda_{0}(l)$. If $C \subset \Lambda_{0}(l)$ is a closed curve then $A^{-1} C \subset T_{0}(l)$ is closed too. Let $z \in \Lambda_{0}(l), w \in T_{0}(l)$, $c=c(w)=\left(a_{31} w_{1}+a_{32} w_{2}+a_{33}\right)^{-1}$, we have:

$$
\begin{aligned}
-\int_{C} \theta & =3 i \int_{C} \partial \ln (-\langle z, z\rangle)=3 i \int_{A^{-1} C} \partial \ln (-\langle A w, A w\rangle) \\
& =3 i \int_{A^{-1} C} \partial \ln (-\langle w, w\rangle c \bar{c})=3 i \int_{A^{-1} C}(\partial \ln (-\langle w, w\rangle)+\partial \ln c) \\
& =3 i \int_{A^{-1} C}(\partial \ln (-\langle w, w\rangle)+d \ln c)=3 i \int_{A^{-1} C} \partial \ln (-\langle w, w\rangle)
\end{aligned}
$$

so $\int_{C} \theta$ is $A^{-1}$-invariant (in fact $S U(2,1)$-invariant) and it is enough to prove that $T_{0}(l)$ satisfies the Bohr-Sommerfeld condition. The restriction of $\theta$ to 
$\tilde{T}_{0}(l)$ is

$$
\begin{aligned}
& -3 i \frac{\bar{w}_{1} d w_{1}+\bar{w}_{2} d w_{2}}{w_{1} \bar{w}_{1}+w_{2} \bar{w}_{2}-1} \\
& =-3 i \frac{\left(1-w_{2} \bar{w}_{2}\right) R^{2} i d \Theta+\sqrt{1-w_{2} \bar{w}_{2}} R^{2} d \sqrt{1-w_{2} \bar{w}_{2}}+\bar{w}_{2} d w_{2}}{\left(1-w_{2} \bar{w}_{2}\right) R^{2}+w_{2} \bar{w}_{2}-1} \\
& =-3 i \frac{\left(1-w_{2}^{2}\right) R^{2} i d \Theta+\sqrt{1-w_{2}^{2}} R^{2} d \sqrt{1-w_{2}^{2}}+w_{2} d w_{2}}{\left(1-w_{2}^{2}\right)\left(R^{2}-1\right)} \\
& =-3 i \frac{\left(1-w_{2}^{2}\right) R^{2} i d \Theta+\sqrt{1-w_{2}^{2}} R^{2} \frac{-2 w_{2} d w_{2}}{2 \sqrt{1-w_{2}^{2}}}+w_{2} d w_{2}}{\left(1-w_{2}^{2}\right)\left(R^{2}-1\right)} \\
& =-3 i\left(\frac{R^{2} i}{R^{2}-1} d \Theta-\frac{w_{2} d w_{2}}{1-w_{2}^{2}}\right)=3 \frac{R^{2}}{R^{2}-1} d \Theta-3 i \frac{1}{2} d \ln \left(1-w_{2}^{2}\right) \\
& =-\frac{l}{k} d \Theta-\frac{3}{2} i d \ln \left(1-w_{2}^{2}\right),
\end{aligned}
$$

then

$$
\begin{aligned}
& \frac{k}{2 \pi} \int_{A^{-1} C}\left(\frac{l}{k} d \Theta+\frac{3}{2} i d \ln \left(1-w_{2}^{2}\right)\right) \\
& =\frac{l}{2 \pi} \int_{A^{-1} C} d \Theta=\frac{l}{2 \pi} 2 \pi m=l m \in \mathbb{Z} .
\end{aligned}
$$

So the torus $\Lambda_{0}(l)$ is a Lagrangian submanifold satisfying the BohrSommerfeld condition.

Proposition 4.5. The orthogonal projection of the delta function at $(w, \eta) \in \tilde{P}$ into $\tilde{E}_{k}$ is

$$
\Psi_{(w, \eta)}(z, \zeta):=\tilde{\Pi}_{k}\left(\delta_{(w, \eta)}\right)=\frac{(3 k-1)(3 k-2)}{4 \pi^{2}} \frac{\zeta^{k} \bar{\eta}^{k}}{\langle z, w\rangle^{3 k}} .
$$

Remark 4.6. The orthogonal projection of the delta function at $(w, \eta) \in \tilde{P}$ into $\tilde{E}_{k}$ is the coherent state in $\tilde{E}_{k}$ associated to the point $(w, \eta) \in \tilde{P}$, by definition $g \Psi_{(w, \eta)}=\Psi_{g(w, \eta)}$ for $g \in S U(2,1)$.

Proof. The fact that $\Psi_{(w, \eta)}=\tilde{\Pi}_{k}\left(\delta_{(w, \eta)}\right)$ is equivalent to the reproducing property:

$$
F(w, \eta)=\int_{\tilde{P}} \bar{\Psi}_{(w, \eta)}(z, \zeta) F(z, \zeta) d V \wedge d \psi
$$


for all $F \in \tilde{E}_{k}$. Given any orthonormal basis $\left\{F_{l, k}\right\}$ for $\tilde{E}_{k}$, we can write the reproducing kernel as the series $\Psi_{(w, \eta)}(z, \zeta)=\sum_{l} \bar{F}_{l, k}(w, \eta) F_{l, k}(z, \zeta)$ which converges absolutely and uniformly on compact sets.

Using the basis

$$
F_{l, m, k}(z, \zeta)=\frac{1}{2 \pi} \sqrt{\frac{(3 k+l+m-1) !}{l ! m !(3 k-3) !}} z_{1}^{l} z_{2}^{m} \zeta^{k}
$$

which is orthonormal with respect to the inner product

$$
\left(f(z) \zeta^{k}, g(z) \zeta^{k}\right)=i^{2} \int_{B^{2}} f \bar{g}(-\langle z, z\rangle)^{3 k-3} d z_{1} \wedge d \bar{z}_{1} \wedge d z_{2} \wedge d \bar{z}_{2}
$$

we obtain:

$$
\begin{aligned}
\Psi_{(w, \eta)}(z, \zeta) & =\sum_{l, m} \bar{F}_{l, m, k}(w, \eta) F_{l, m, k}(z, \zeta) \\
& =\sum_{l, m} \frac{1}{(2 \pi)^{2}} \frac{(3 k+l+m-1) !}{l ! m !(3 k-3) !} \bar{w}_{1}^{l} \bar{w}_{2}^{m} z_{1}^{l} z_{2}^{m} \zeta^{k} \bar{\eta}^{k} \\
& =\frac{\zeta^{k} \bar{\eta}^{k}}{4 \pi^{2}(3 k-3) !} \sum_{l, m} \frac{(3 k+l+m-1) !}{l ! m !}\left(\bar{w}_{1} z_{1}\right)^{l}\left(\bar{w}_{2} z_{2}\right)^{m}
\end{aligned}
$$

To calculate

$$
\sum_{l, m} \frac{(3 k+l+m-1) !}{l ! m !} x^{l} y^{m}=\sum_{m} \frac{y^{m}}{m !} \sum_{l} \frac{(3 k+l+m-1) !}{l !} x^{l}
$$

we notice that

$$
\begin{aligned}
\sum_{l} \frac{(N+l) !}{l !} t^{l} & =\frac{d^{N}}{d t^{N}} \sum_{l=0}^{\infty} t^{l+N}=\frac{d^{N}}{d t^{N}} \frac{t^{N}}{1-t} \\
& =\frac{d^{N}}{d t^{N}}\left(\frac{t^{N}-1}{1-t}+\frac{1}{1-t}\right) \\
& =\frac{d^{N}}{d t^{N}} \frac{1}{1-t}=\frac{N !}{(1-t)^{N+1}}
\end{aligned}
$$


hence

$$
\begin{aligned}
\sum_{m} \frac{y^{m}}{m !} \sum_{l} \frac{(3 k+l+m-1) !}{l !} x^{l} & =\sum_{m} \frac{y^{m}}{m !} \frac{(3 k+m-1) !}{(1-x)^{3 k+m}} \\
& =\frac{1}{(1-x)^{3 k}} \sum_{m} \frac{(3 k+m-1) !}{m !}\left(\frac{y}{1-x}\right)^{m} \\
& =\frac{1}{(1-x)^{3 k}} \frac{(3 k-1) !}{\left(1-\frac{y}{1-x}\right)^{3 k}}=\frac{(3 k-1) !}{(1-x-y)^{3 k}},
\end{aligned}
$$

so

$$
\begin{aligned}
\Psi_{(w, \eta)}(z, \zeta) & =\frac{\zeta^{k} \bar{\eta}^{k}}{4 \pi^{2}(3 k-3) !} \frac{(3 k-1) !}{\left(1-\bar{w}_{1} z_{1}-\bar{w}_{2} z_{2}\right)^{3 k}} \\
& =\frac{(3 k-1)(3 k-2)}{4 \pi^{2}} \zeta^{k} \bar{\eta}^{k} \frac{1}{(-\langle z, w\rangle)^{3 k}}
\end{aligned}
$$

For $\tilde{E}_{2 k}$ we have:

$$
\Psi_{(u, \eta)}(z, \zeta)=\tilde{\Pi}_{2 k}\left(\delta_{(u, \eta)}\right)=\frac{(6 k-1)(6 k-2)}{4 \pi^{2}} \frac{\zeta^{2 k} \bar{\eta}^{2 k}}{\langle z, u\rangle^{6 k}} .
$$

We omit the weight in the notation $\Psi_{(u, \eta)}(z, \zeta)$ but further exposition will be for $E_{2 k}$ (i.e., weight $6 k$ ) so this will not lead to any confusion.

To get the orthogonal projection of the delta function at $[(u, \eta)] \in P=$ $\Gamma \backslash \tilde{P}$ (by $[(u, \eta)]$ we denote the equivalence class of $(u, \eta))$ into $E_{2 k}$ we average over the action of $\Gamma$ :

$$
\Pi_{2 k}\left(\delta_{[(u, \eta)]}\right)=\sum_{g \in \Gamma} g \Psi_{(u, \eta)} .
$$

The series (7) converges absolutely and uniformly on compact sets by Theorem 9.1 [4].

Following the method of [6], to the submanifold $\Lambda(l) \subset P$ we associate a section $u_{2 k} \in E_{2 k}$ defined as follows:

$$
\begin{aligned}
u_{2 k} & =\int_{\Lambda(l)} \Pi_{2 k}\left(\delta_{[(u, \eta)]}\right) \nu=\sum_{g \in \Gamma} \int_{\Lambda(l)} g \Psi_{(u, \eta)} \nu \\
& =\sum_{g \in \Gamma / \Gamma_{0}} \sum_{m=-\infty}^{+\infty} \int_{\Lambda(l)} g \gamma_{0}^{m} \Psi_{(u, \eta)}(z, \zeta) \nu
\end{aligned}
$$




$$
\begin{aligned}
& =\sum_{g \in \Gamma / \Gamma_{0}} \sum_{m=-\infty}^{+\infty} \int_{\Lambda(l)} g \Psi_{\gamma_{0}^{m}(u, \eta)}(z, \zeta) \nu \\
& =\sum_{g \in \Gamma / \Gamma_{0}} g \int_{\tilde{\Lambda}(l)} \Psi_{(u, \eta)}(z, \zeta) \nu=\sum_{g^{-1} \in \Gamma_{0} \backslash \Gamma} g^{-1} \int_{\tilde{\Lambda}(l)} \Psi_{(u, \eta)}(z, \zeta) \nu
\end{aligned}
$$

where

$$
\nu=\frac{d\left(A^{-1} u_{1}\right)}{A^{-1} u_{1}} \wedge \frac{d\left(A^{-1} u_{2}\right)}{1-\left(A^{-1} u_{2}\right)^{2}} .
$$

\section{Proposition 4.7.}

(i) $I:=\int_{\tilde{\Lambda}(l)} \Psi_{(u, \eta)}(z, \zeta) \nu=C \frac{\langle z, v\rangle^{2 l}}{(\langle z, X\rangle\langle z, Y\rangle)^{3 k+l}} \zeta^{2 k}$, where the constant $C$ is given by

$$
C=\frac{2^{3 k+l-2}}{\pi} \frac{((3 k+l-1) !)^{2}}{(2 l) !(6 k-3) !} \frac{(3 k)^{3 k} l^{l}}{(3 k+l)^{3 k+l}}(-\langle Y, X\rangle)^{3 k+l}
$$

(ii) $\int_{\Lambda(l)}|\nu|=2 \pi \ln |\lambda|$.

Remark 4.8. The 2 -form $\nu$ on $\tilde{\Lambda}(l)$ is $\gamma_{0}$-invariant and in properly chosen coordinates $(r, \Theta)$ on $\tilde{\Lambda}_{0}(l)$ it is expressed as $\nu=\frac{i}{2} d \Theta \wedge \frac{d r}{r}$.

Proof. Let $u \in \tilde{\Lambda}(l), w=A^{-1} u \in \tilde{T}(l)$, then

$$
\begin{aligned}
I= & \frac{(6 k-1)(6 k-2)}{4 \pi^{2}} \zeta^{2 k} \int_{\tilde{\Lambda}(l)} \frac{\bar{\eta}^{2 k}}{\langle z, u\rangle^{6 k}} \frac{d\left(A^{-1} u_{1}\right)}{A^{-1} u_{1}} \wedge \frac{d\left(A^{-1} u_{2}\right)}{1-\left(A^{-1} u_{2}\right)^{2}} \\
= & \frac{(6 k-1)(6 k-2)}{4 \pi^{2}} \zeta^{2 k} \\
& \cdot \int_{\tilde{T}(l)} \frac{\left((-\langle w, w\rangle)^{\frac{3}{2}} e^{-i \psi} \operatorname{det} \bar{J}(A, w)\right)^{2 k}}{\langle z, A w\rangle^{6 k}} \frac{d w_{1}}{w_{1}} \wedge \frac{d w_{2}}{1-w_{2}^{2}} \\
= & \frac{(6 k-1)(6 k-2)}{4 \pi^{2}} \zeta^{2 k} \int_{\tilde{T}(l)} \frac{(-\langle w, w\rangle)^{3 k} e^{-i 2 k \psi}(\operatorname{det} \bar{J}(A, w))^{2 k}}{\left\langle A^{-1} z, w\right\rangle^{6 k}(\operatorname{det} \bar{J}(A, w))^{2 k}} \\
& \cdot\left(\operatorname{det} J\left(A^{-1}, z\right)\right)^{2 k} \frac{d w_{1}}{w_{1}} \wedge \frac{d w_{2}}{1-w_{2}^{2}},
\end{aligned}
$$

let $A^{-1} z=\left(\begin{array}{c}v_{1} \\ v_{2} \\ 1\end{array}\right)$, then we get:

$$
I=\frac{(6 k-1)(6 k-2)}{4 \pi^{2}} \zeta^{2 k}\left(\operatorname{det} J\left(A^{-1}, z\right)\right)^{2 k}
$$




$$
\int_{\tilde{T}(l)} \frac{(-\langle w, w\rangle)^{3 k} e^{-i 2 k \psi}}{\left(v_{1} \bar{w}_{1}+v_{2} \bar{w}_{2}-1\right)^{6 k}} \frac{d w_{1}}{w_{1}} \wedge \frac{d w_{2}}{1-w_{2}^{2}}
$$

on $\tilde{T}(l)$

$$
\begin{aligned}
& w_{2}=\frac{r-1}{r+1}, w_{1}=\sqrt{1-w_{2}^{2}} R e^{i \Theta}=\frac{2 \sqrt{r}}{r+1} R e^{i \Theta} \\
& -\langle w, w\rangle=\left(1-R^{2}\right)\left(1-w_{2}^{2}\right)=\left(1-R^{2}\right) \frac{4 r}{(r+1)^{2}}
\end{aligned}
$$

so we have:

$$
\begin{aligned}
I= & \frac{(6 k-1)(6 k-2)}{4 \pi^{2}} \zeta^{2 k}\left(\operatorname{det} J\left(A^{-1}, z\right)\right)^{2 k}\left(4\left(1-R^{2}\right)\right)^{3 k} \\
& \cdot \int_{\tilde{T}(l)} \frac{\left(\frac{r}{(r+1)^{2}}\right)^{3 k} e^{-i 2 k \psi}}{\left(v_{1} \frac{2 \sqrt{r}}{r+1} R e^{-i \Theta}+v_{2} \frac{r-1}{r+1}-1\right)^{6 k}} \frac{i}{2} d \Theta \wedge \frac{d r}{r} \\
= & \frac{(6 k-1)(6 k-2)}{4 \pi^{2}} \zeta^{2 k}\left(\operatorname{det} J\left(A^{-1}, z\right)\right)^{2 k}\left(4\left(1-R^{2}\right)\right)^{3 k} \frac{i}{2} \\
& \cdot \int_{0}^{\infty} d r \int_{0}^{2 \pi} d \Theta \frac{r^{3 k-1} e^{i 2 l \Theta}}{\left(v_{1} 2 \sqrt{r} R e^{-i \Theta}+v_{2}(r-1)-r-1\right)^{6 k}} .
\end{aligned}
$$

The integral

$$
\int_{|w|=1} \frac{1}{(A w+B)^{6 k}} \frac{d w}{w^{2 l+1}}, \quad\left|\frac{B}{A}\right|>1
$$

is equal to

$$
\left.\frac{2 \pi i}{(2 l) !} \frac{d^{2 l}}{d w^{2 l}} \frac{1}{(A w+B)^{6 k}}\right|_{w=0}=\frac{2 \pi i}{(2 l) !} \frac{(6 k+2 l-1) !}{(6 k-1) !} \frac{A^{2 l}}{B^{6 k+2 l}}
$$

Let $w=e^{-i \Theta}, A=v_{1} 2 \sqrt{r} R, B=v_{2}(r-1)-r-1$. Let us check that $\left|\frac{B}{A}\right|>1$.

$$
\begin{aligned}
\left|\frac{v_{2}(r-1)-r-1}{v_{1} 2 \sqrt{r} R}\right| & =\left|\frac{v_{2} w_{2}-1}{v_{1} R \sqrt{1-w_{2}^{2}}}\right|>\frac{\left|v_{2} w_{2}-1\right|}{\sqrt{1-v_{2} \bar{v}_{2}} R \sqrt{1-w_{2}^{2}}} \\
& \geq \frac{\left|v_{2} w_{2}-1\right|}{\sqrt{1-v_{2} \bar{v}_{2}} \sqrt{1-w_{2}^{2}}} \geq 1
\end{aligned}
$$

because

$$
\begin{aligned}
0 & \leq\left|v_{2}-w_{2}\right|^{2}=\left(\bar{v}_{2}-w_{2}\right)\left(v_{2}-w_{2}\right)=v_{2} \bar{v}_{2}-\bar{v}_{2} w_{2}-w_{2} v_{2}+w_{2}^{2} \\
& =-\bar{v}_{2} w_{2}-w_{2} v_{2}+v_{2} \bar{v}_{2} w_{2}^{2}+1+v_{2} \bar{v}_{2}+w_{2}^{2}-v_{2} \bar{v}_{2} w_{2}^{2}-1
\end{aligned}
$$




$$
=\left(\bar{v}_{2} w_{2}-1\right)\left(v_{2} w_{2}-1\right)-\left(1-v_{2} \bar{v}_{2}\right)\left(1-w_{2}^{2}\right) .
$$

We get:

$$
\begin{aligned}
I= & -\frac{(6 k-1)(6 k-2)}{4 \pi^{2}} \zeta^{2 k}\left(4\left(1-R^{2}\right)\right)^{3 k} \frac{i}{2} \frac{2 \pi i}{(2 l) !} \frac{(6 k+2 l-1) !}{(6 k-1) !} \\
& \cdot\left(\operatorname{det} J\left(A^{-1}, z\right)\right)^{2 k} \int_{0}^{\infty} \frac{r^{3 k-1+l} v_{1}^{2 l} 2^{2 l} R^{2 l}}{\left(v_{2}(r-1)-r-1\right)^{6 k+2 l}} d r \\
= & \frac{4^{3 k-1+l}}{\pi} \frac{(6 k+2 l-1) !}{(2 l) !(6 k-3) !} R^{2 l}\left(1-R^{2}\right)^{3 k} \zeta^{2 k}\left(\operatorname{det} J\left(A^{-1}, z\right)\right)^{2 k} \\
& \cdot \frac{v_{1}^{2 l}}{\left(v_{2}-1\right)^{6 k+2 l}} \int_{0}^{\infty} \frac{r^{3 k-1+l}}{\left(r-\frac{v_{2}+1}{v_{2}-1}\right)^{6 k+2 l}} d r .
\end{aligned}
$$

Notice that $\frac{v_{2}+1}{v_{2}-1}$ can not be a real non-negative number, and the integration by parts gives:

$$
\int_{0}^{\infty} \frac{r^{3 k-1+l}}{\left(r-\frac{v_{2}+1}{v_{2}-1}\right)^{6 k+2 l}} d r=\frac{((3 k+l-1) !)^{2}}{(6 k+2 l-1) !}\left(-\frac{v_{2}-1}{v_{2}+1}\right)^{3 k+l},
$$

hence

$$
\begin{aligned}
& I=\frac{4^{3 k+l-1}}{\pi} \frac{((3 k+l-1) !)^{2}}{(2 l) !(6 k-3) !} R^{2 l}\left(1-R^{2}\right)^{3 k} \\
& \cdot\left(\operatorname{det} J\left(A^{-1}, z\right)\right)^{2 k} \frac{v_{1}^{2 l} \zeta^{2 k}}{\left[\left(1-v_{2}\right)\left(1+v_{2}\right)\right]^{3 k+l}}
\end{aligned}
$$

and

$$
\begin{aligned}
& \frac{v_{1}^{2 l}}{\left[\left(1-v_{2}\right)\left(1+v_{2}\right)\right]^{3 k+l}} \\
& =\frac{\left\langle A^{-1} z, A^{-1} \cdot v\right\rangle^{2 l}}{\left(\left\langle A^{-1} z, A^{-1} X\right\rangle\left\langle A^{-1} z, A^{-1} Y\right\rangle\right)^{3 k+l}} \\
& =\frac{\langle z, v\rangle^{2 l}}{(\langle z, X\rangle\langle z, Y\rangle)^{3 k+l}}\left(\operatorname{det} J\left(A^{-1}, z\right)\right)^{-2 k}\left(-\frac{\langle Y, X\rangle}{2}\right)^{3 k+l},
\end{aligned}
$$

therefore

$$
I=\frac{2^{3 k+l-2}}{\pi} \frac{((3 k+l-1) !)^{2}}{(2 l) !(6 k-3) !} \frac{(3 k)^{3 k} l^{l}}{(3 k+l)^{3 k+l}}(-\langle Y, X\rangle)^{3 k+l} \frac{\langle z, v\rangle^{2 l}}{(\langle z, X\rangle\langle z, Y\rangle)^{3 k+l}} \zeta^{2 k} .
$$

Proof of (ii):

$$
\int_{\Lambda(l)} \nu=\int_{A T(l)}\left|\frac{d\left(A^{-1} u_{1}\right)}{A^{-1} u_{1}} \wedge \frac{d\left(A^{-1} u_{2}\right)}{1-\left(A^{-1} u_{2}\right)^{2}}\right|
$$




$$
=\int_{T(l)}\left|\frac{d w_{1}}{w_{1}} \wedge \frac{d w_{2}}{1-w_{2}^{2}}\right|=\int_{|\lambda|^{-1}}^{|\lambda|} d r \int_{0}^{2 \pi} d \Theta \frac{1}{2 r}=2 \pi \ln |\lambda| .
$$

We got:

$$
u_{2 k}(z, \zeta)=\zeta^{2 k} \sum_{g \in \Gamma_{0} \backslash \Gamma} q_{l}(g z)(\operatorname{det} J(g, z))^{2 k} \in \tilde{S}_{2(n+1) k}(\Gamma)
$$

where

$$
q_{l}(z)=C \frac{\langle z, v\rangle^{2 l}}{(\langle z, X\rangle\langle z, Y\rangle)^{3 k+l}}
$$

and the relative Poincare series associated to $\Lambda_{0}(l)$ is

$$
\Theta_{\gamma_{0}, l, k}(z):=\sum_{g \in \Gamma_{0} \backslash \Gamma} q_{l}(g z)(\operatorname{det} J(g, z))^{2 k} \in S_{2(n+1) k} .
$$

From the results of [6] (Theorem 3.2, Corollary 3.3) it follows that for large values of $k$

$$
\left.\left\|u_{2 k}\right\|^{2}=\left\|\Theta_{\gamma_{0}, l, k}\right\|^{2} \sim \frac{2 k}{\pi} \int_{\Lambda(l)}|\nu|=4 k \ln |\lambda|\right),
$$

and, in particular, the relative Poincaré series $\Theta_{\gamma_{0}, l, k}$ are not identically zero for large weights.

Acknowledgements. The author would like to thank Svetlana Katok for suggesting to consider the problem and the referee for useful and interesting comments.

\section{Appendix A.}

We shall prove the following theorem modifying the proof of convergence of Poincaré series contained in [4] and [3].

Theorem A.1. Let $\varphi$ be a $\mathbb{C}$-valued function on $G=S U(n, 1)$. Assume that

1) $\varphi$ is $Z(\mathfrak{g})$-finite,

2) $\varphi \in L^{1}\left(\Gamma_{0} \backslash G\right)$, 
3) $\varphi$ is K-finite on the right.

Let $p_{\varphi}(x)=\sum_{\gamma \in \Gamma_{0} \backslash \Gamma} \varphi(\gamma x)$.

Then $p_{\varphi}$ converges absolutely and uniformly on compact sets.

Proof. By Lemma 9.2 [4] there exists $\alpha \in C_{c}^{\infty}(G)$ satisfying $\alpha\left(k^{-1} x k\right)=$ $\alpha(x), k \in K, x \in G$, such that $\varphi=\varphi * \alpha$. Fix a neighborhood $U$ of 1 in $G$ such that $U^{-1}=U$, the closure of $U$ is compact, and $U \supset \operatorname{supp} \alpha$. We have:

$$
\varphi(\gamma x)=(\varphi * \alpha)(\gamma x)=\int_{G} \varphi(\gamma x y) \alpha\left(y^{-1}\right) d y=\int_{U} \varphi(\gamma x y) \alpha\left(y^{-1}\right) d y
$$

hence

$$
|\varphi(\gamma x)| \leq\|\alpha\|_{\infty} \int_{U}|\varphi(\gamma x y)| d y=\|\alpha\|_{\infty} \int_{x U}|\varphi(\gamma y)| d y
$$

Here $\|\alpha\|_{\infty}=\sup _{y \in U}|\alpha(y)|$.

Fix a compact subset $C$ of $G$. We want to prove absolute and uniform convergence on $C$. The closure of $C U$ is compact. $C U$ is covered by $N$ copies of a fundamental domain of $\Gamma$ in $G$ (i.e., a connected set of representatives of $\Gamma \backslash G)$, where $N$ is a positive integer. Denote these domains by $F_{1}, \ldots, F_{N}$.

Let $x \in C$. Then

$$
|\varphi(\gamma x)| \leq\|\alpha\|_{\infty} \int_{x U}|\varphi(\gamma y)| d y \leq\|\alpha\|_{\infty} \int_{C U}|\varphi(\gamma y)| d y
$$

and we get

$$
\begin{aligned}
& \sum_{\gamma \in \Gamma_{0} \backslash \Gamma}\|\alpha\|_{\infty} \int_{C U}|\varphi(\gamma y)| d y \\
= & \|\alpha\|_{\infty} \sum_{\gamma \in \Gamma_{0} \backslash \Gamma} \int_{C U}|\varphi(\gamma y)| d y \\
\leq & \|\alpha\|_{\infty} \sum_{\gamma \in \Gamma_{0} \backslash \Gamma}\left(\int_{F_{1}}|\varphi(\gamma y)| d y+\cdots+\int_{F_{N}}|\varphi(\gamma y)| d y\right) \\
= & \|\alpha\|_{\infty}\left(\sum_{\gamma \in \Gamma_{0} \backslash \Gamma} \int_{F_{1}}|\varphi(\gamma y)| d y+\cdots+\sum_{\gamma \in \Gamma_{0} \backslash \Gamma} \int_{F_{N}}|\varphi(\gamma y)| d y\right) \\
= & N\|\alpha\|_{\infty} \int_{\Gamma_{0} \backslash G}|\varphi(y)| d y<\infty .
\end{aligned}
$$


So we proved that

$$
|\varphi(\gamma x)| \leq c_{\gamma}:=\|\alpha\|_{\infty} \int_{C U}|\varphi(\gamma y)| d y
$$

and that the numerical series $\sum_{\gamma \in \Gamma_{0} \backslash \Gamma} c_{\gamma}$ converges, hence by Weierstrass theorem the series $\sum_{\gamma \in \Gamma_{0} \backslash \Gamma} \varphi(\gamma x)$ converges absolutely and uniformly on $C$.

\section{References.}

[1] F. Berezin, General concept of quantization, Comm. Math. Phys., 40 (1975), 153-174.

[2] M. Bordemann, E. Meinrenken, and M. Schlichenmaier, Toeplitz quantization of Kähler manifolds and $g l(N), N \rightarrow \infty$ limits, Comm. Math. Phys., 165(2) (1994), 281-296.

[3] A. Borel, Automorphic forms on $S L_{2}(\mathbb{R})$, Cambridge University Press, 1997.

[4] A. Borel, Introduction to automorphic forms, Proc. Symp. Pure Math., 9 (1966), 199-210.

[5] D. Borthwick, A. Lesniewski, and H. Upmeier, Non-perturbative deformation quantization of Cartan domains, J. Funct. Anal., 113 (1993), 153-176.

[6] D. Borthwick, T. Paul, and A. Uribe, Legendrian distributions with applications to relative Poincaré series, Invent. Math., 122 (1995), 359402 .

[7] L. Boutet de Monvel and V. Guillemin, The spectral theory of Toeplitz operators, Princeton University Press, 1981.

[8] D. Epstein, Complex hyperbolic geometry, in 'Analytical and geometric aspects of hyperbolic space,' London Math. Soc. Lecture Note Ser., 111, Cambridge Univ. Press, 1987.

[9] T. Foth and S. Katok, Spanning sets for automorphic forms and dynamics of the frame flow on complex hyperbolic spaces, to appear in Ergod. Th. Dyn. Sys. 
[10] W. Goldman, Complex hyperbolic geometry, Oxford University Press, 1999.

[11] V. Guillemin and S. Sternberg, Geometric asymptotics, Math. Surveys, 14, AMS, 1977.

[12] N. Hurt, Geometric quantization in action, D. Reidel Publ. Co., 1983.

[13] S. Klimek and A. Lesniewski, Quantum Riemann surfaces I. The unit disc, Comm. Math. Phys., 146 (1992), 103-122.

[14] S. Klimek and A. Lesniewski, Quantum Riemann surfaces II. The discrete series, Lett. Math. Phys., 24 (1992), 125-139.

[15] S. Kobayashi, C. Horst, and H. Wu, Complex differential geometry, Birkhäuser, 1983.

[16] J. Kollar, Shafarevich maps and automorphic forms, Princeton University Press, 1995.

[17] S. Krantz, Function theory of several complex vaiables, Wadsworth \& Brooks/Cole, 1992.

[18] A. Perelomov, Generalized cohereht states and applications, SpringerVerlag, 1986.

[19] J. Rawnsley, Quantization on Kähler manifolds, in 'Differential geometric methods in theoretical physics,' Lect. Notes in Phys., 375 (1991), 155-161.

[20] J. Rawnsley, Deformation quantization of Kähler manifolds, in 'Symplectic geometry and mathematical physics,' Prog. in Math., 99 (1991), 366-373.

[21] Y. Tai and H. Resnikoff, On the structure of a graded ring of automorphic forms on the 2-dimensional complex ball, I and II, Math. Ann., 238 (1978), 97-117; Math. Ann., 258 (1982), 367-382.

[22] J. Sniatycki, Geometric quantization and quantum mechanics, SpringerVerlag, 1980.

[23] D. Zhelobenko and A. Shtern, Representations of Lie groups (Russian), "Nauka", Moscow, 1983. 
[24] N. Woodhouse, Geometric quantization, Oxford University Press, 1980.

Department of Mathematics, University of Michigan

Ann Arbor, MI 48109

E-mail address: foth@umich.edu

Received November 16, 2000. 